\title{
NEURO-ONCOLOGY
}

\section{Antifungal treatment for glioma?}

Microglia and CNS-specific macrophages are innate immune cells that reside in the brain. However, when faced with a glioma, instead of fighting, these monocytoid cells have their normal function suppressed and are co-opted into tumour proliferation, which makes treating cancers of the brain extremely difficult. A new report published in Nature Neuroscience describes a mechanism by which monocytoid cells can be reactivated to fight back against the cancer and might provide a new therapeutic treatment for glioma.

"The neoplastic growth of malignant brain tumours is maintained by stem-like cells called glioma stem cells or brain tumour-initiating cells (BTICs). There have been no effective ways to control the growth of BTICs, so we inquired whether the nontumour cells within the CNS could be recruited to locally affect BTICs," explains Voon Wee Yong from the University of Calgary, Canada, who led the study.

In culture, BTICs proliferate into large aggregated clumps known as neurospheres and, when transplanted into mouse brains, these cells are potent stimulators of gliomas. The researchers exploited this ability to investigate the relationship between brain-specific immune cells and gliomas. The team demonstrated that monocytoid cells from human patients without glioma can reduce the size of neurospheres and BTIC-induced tumours in mouse brains. However, when taken from patients with a terminal glioma, the monocytoids had no effect on the neurospheres or tumours. "Our study has provided novel data on the interactions, mechanisms and functions between microglia and macrophages with BTICs, which was for the most part unknown in the context of BTIC biology," highlights Yong.

The team also screened $>1,000$ different compounds for substances that might reactivate monocytoid cells and identified amphotericin $B$ $(\mathrm{AmpB})$, which induced expression of activated microglia markers. AmpB is commonly used as an antifungal treatment in humans, but microglia stimulated with AmpB reduced neurosphere formation of BTICs in culture. When tested on BTIC-induced gliomas in mice, AmpB reduced tumour size and extended the life of the animals and, strikingly, enabled monocyoid cells from human patients with glioma to reduce BTIC-induced tumours in mice.

The researchers hope AmpB might be used to treat glioma in a clinical setting. "Although $\mathrm{AmpB}$ is a medication with considerable adverse effects when used at high doses to treat life-threatening fungal infections, we have used a dose that provides serum concentrations of drug that is 10 -times lower than that encountered in the treatment of human fungal infections," clarifies Yong. "Indeed, we encountered no evidence of toxicity with AmpB in our mouse work."

Interestingly, $\mathrm{AmpB}$ was previously associated with the remission and clinical improvement of glioma in a human patient who was prescribed the drug to treat an unrelated fungal infection-sometimes scientific discoveries are serendipitous.

\section{Tim Geach}

This article is modified from the original in Nat. Rev. Clin. Onc. (doi:10.1038/ nrclinonc.2013.249). 